\title{
TRAFFIC MANEUVER PROBLEMS AND CRASHES OF YOUNG DRIVERS
}

\author{
Adam Kirk \\ Nikiforos Stamatiadis \\ Dept. of Civil Engineering \\ University of Kentucky \\ Lexington, KY 40506-0281 \\ E-mail: akirk@ch2m.com \\ E-mail: nstamat@engr.uky.edu
}

\begin{abstract}
While over the past decades the population of younger drivers has been decreasing, their crash rates have increased. Past research has associated their higher crash rates to societal influences and youthful behavior. The objective of this research is to identify the specific driving maneuvers whose unsuccessful undertaking results in specific types of crashes involving these drivers. Four types of crashes were identified as the most prominent for young drivers including crashes at intersections, rear end, crashes resulting from passing maneuvers and single vehicle crashes. The analysis was performed examining the Kentucky crash database for the 1994-1996 period using the quasi-induced exposure method. The results showed that for all crashes there is a general trend of decreasing involvement with increasing age, which indicates that their inexperience is the largest single contributor to their increased crash rates. Of significance is the fact that for all crashes a dramatic decrease of involvement after the first year of driving between the years of 16 and 17 is observed. This may be indicative of a steep learning curve in the first years of driving regarding the ability to control a vehicle. Therefore, very little can be made to improve this phenomenon. Increasing the level of awareness among young drivers about these issues and their likely crash involvement seems to be the only viable approach. However, preliminary efforts from the graduated license show that some of these trends seem to be reduced indicating a possible impact on the crash rates of young drivers.
\end{abstract}

